\title{
Features Preference using Conjoint Analysis Method for E-marketplace Social Care System
}

\author{
Angelina Ervina Jeanette Egeten ${ }^{*}, 1$, Harjanto Prabowo ${ }^{2}$, Ford Lumban $\mathrm{Gaol}^{3}$, Meyliana ${ }^{4}$ \\ ${ }^{1}$ Information Systems Department, Doctor of Computer Science, Bina Nusantara University, Jakarta, 11530, Indonesia \\ ${ }^{2}$ Management Department, BINUS Business School Undergraduate Program, Bina Nusantara University, Jakarta, 11530, Indonesia \\ ${ }^{3}$ Computer Science Department, BINUS Graduate Program, Bina Nusantara University, Jakarta, 11530, Indonesia \\ ${ }^{4}$ Information Systems Department, School of Information System, Bina Nusantara University, Jakarta, 11530, Indonesia
}

\begin{tabular}{l} 
A R T I C L E I N F O \\
\hline Article history: \\
Received: 16 September, 2020 \\
Accepted: 10 December, 2020 \\
Online: 25 December, 2020 \\
\hline Keywords: \\
Features Priority \\
E-marketplace Social Care \\
Intention to Use \\
Customer Satisfaction \\
Conjoint Analysis
\end{tabular}

\section{Introduction}

E-marketplace Social Care is an online business using the services of domestic workers who help users to meet their needs. One of user needs is the support of features which make it easier for user to transact [1]. The problem or the gap in this research is that the intensity of application usage decreases judging by the application rating given by the user each time they perform a transaction activity by the user [2], this is because of the company initially make the features based on their urge without further investigating on user satisfaction level and user experience [3] on similar social care e-marketplace, this causes users feel dissatisfied in using the company application system because they are considered not focus on improving the feature functionality in the

${ }^{*}$ Corresponding Author Angelina Egeten, Email: angelina.egeten@binus.ac.id www.astesj.com

https://dx.doi.org/10.25046/aj0506190 same business, and this led a reason why it is important to decide the feature preferences in the application development of emarketplace social care to meet their needs and help companies to increase user satisfaction on used feature functionality. Previous research supports the importance of assessing user satisfaction rating for information technology artifacts in this case is a feature of the whole system as an incentive for consumers to use the system intensely or loyal [2]. Other studies also support the assessment of user satisfaction with user experience in using the features of application system, where by the result of feature assessment provided by user can help companies to improve feature functionality on the company's application system [3]. The purposes of this study are to find out the feature preferences that will be used in the e-marketplace social care and to find out the 
actual result of user opinion related to the feature preferences found. Thus, this research will answer the research questions about:

- $\quad$ Research Question 1 : what are the feature preferences in emarketplace social care?

- Research Question 2 : are the result of the respondent opinion pattern much different from the result of the respondent actual opinion?

\section{Theoretical Background}

\subsection{E-marketplace Social Care}

E-marketplace is a place where sellers and buyers are brought together in the market demand and supply with various types of products and services that available and provide added value to companies [4]. E-marketplace acts as a third-party that brings together sellers and buyers to get the desired choices and at the same time reduces transaction cost in terms of products and services used [5].

E-marketplace social care provides a household services or health social care which aims to help users to meet with workers in helping meet the needs of the users themselves [6], where these workers are under the distribution agencies who cooperate with emarketplace companies.

\subsection{Customer Satisfaction}

In general, customer satisfaction depends on the services used by users, as well as the purchased products. If users are satisfied with the products and services, user satisfaction is fulfilled, or the service exceeds user expectations, the user is very happy and satisfied. Likewise, if the service does not match the user's expectations, the user is not satisfied [7].

Customer Satisfaction is not only based on the user's assessment of the products and services used, but also on the user experience in using service application features and the system's ability to convey complete service information [3] and has an innovative feature [2].

\subsection{Intention to Use}

It is very important to maintain the intensity of reuse the services used in meeting user needs, because when users need the service they will always predict or surely use the service when they need assistance in meeting their needs [1]. Not only in terms of the offered services, but the system support of the services is also the main reason for users to use the services because it can be used anywhere, anytime and are supported with innovative features [2].

\section{Research Method}

This study uses a conjoint analysis method to determine the features preference used by users in the e-marketplace social care system. This method was chosen to evaluate the user's features preference of the services used [8] [9] and be able to produce accurate answer predictions that did not much different between the respondent's opinion pattern and the respondent's actual opinion. Conjoint analysis can be expressed in the following model as a part of the multivariate dependence method, namely [10]:

$$
\mathrm{Y}_{1}=\mathrm{X}_{1}+\mathrm{X}_{2}+\mathrm{X}_{3}+\ldots .+\mathrm{X}_{\mathrm{n}}
$$

where:

$\mathrm{X}=$ Independence variables are often referred as factors and a non-metric data part of the factor called levels.

$Y_{1}=$ The overall opinion of respondents on the various factor and levels of a product or service.

The conjoint process is carried out to categorize the emarketplace social care features against the functional attributes of the e-marketplace social care, which consist of Identity, Conversation, Information and Service, Reputation, dan Sharing. The process produces preference features for each functionality, so that each functionality has a feature that represent in need.

The population in this study was users of e-marketplace social care services in Indonesia where the data is collected from JanuaryFebruary 2020. 521 respondents are used as sample where the questionnaire is distributed using google docs and paper directly filled by users who already had experience in using similar emarketplace social care applications. Users are asked to rate the 25 preferences that have been set out in the questionnaire based on the results of random features of the orthoplan using SPSS version 23. The questionnaire result that has been filled in by the user is collected and tabulated, then the data tested for validity and conjoint analysis to answer the research questions in accordance with the purpose of this study.

\section{Result and Discussion}

The following are the steps in conjoint analysis, namely:

\subsection{E-marketplace Social Care Feature Preferences Based on}

\section{Similar Application}

Based on the feature functionality equation of 4 other similar e-marketplace social care applications that have been selected, they are mapped into the social care application functionality, which will be used for conjoint analysis test to identify the preferred features of the e-marketplace social care system.

Table 1: Feature Preferences of E-marketplace Social Care

\begin{tabular}{|c|c|c|c|c|c|}
\hline Feature & $\begin{array}{c}\text { Functionality } \\
\text { Social Care } \\
\text { Application }\end{array}$ & $\begin{array}{c}\text { App } \\
\text { A }\end{array}$ & $\begin{array}{c}\text { App } \\
\text { B }\end{array}$ & $\begin{array}{c}\text { App } \\
\text { C }\end{array}$ & $\begin{array}{c}\text { App } \\
\text { D }\end{array}$ \\
\hline Photo & Identity & $\checkmark$ & - & - & - \\
\hline $\begin{array}{c}\text { Sign Up } \\
\text { Sign Up) }\end{array}$ & Identity & $\checkmark$ & $\checkmark$ & $\checkmark$ & $\checkmark$ \\
\hline $\begin{array}{c}\text { Account } \\
\text { Profile }\end{array}$ & Identity & $\checkmark$ & $\checkmark$ & $\checkmark$ & $\checkmark$ \\
\hline $\begin{array}{c}\text { Forgot } \\
\text { Password }\end{array}$ & Identity & $\checkmark$ & $\checkmark$ & $\checkmark$ & $\checkmark$ \\
\hline $\begin{array}{c}\text { Services } \\
\text { Assistance } \\
\text { Chat }\end{array}$ & Conversation & $\checkmark$ & $\checkmark$ & $\checkmark$ & $\checkmark$ \\
\hline Testimonial & Conversation & $\checkmark$ & $\checkmark$ & $\checkmark$ & $\checkmark$ \\
\hline Like (Like) & Conversation & $\checkmark$ & - & - & - \\
\hline $\begin{array}{c}\text { Special } \\
\text { Request }\end{array}$ & Service & $\checkmark$ & - & - & - \\
\hline $\begin{array}{c}\text { List of } \\
\text { Services \& } \\
\text { Detail of } \\
\text { Services }\end{array}$ & Service & $\checkmark$ & $\checkmark$ & $\checkmark$ & $\checkmark$ \\
\hline $\begin{array}{c}\text { Order and } \\
\text { Order } \\
\text { History }\end{array}$ & Service & $\checkmark$ & $\checkmark$ & $\checkmark$ & $\checkmark$ \\
\hline
\end{tabular}




\begin{tabular}{|c|c|c|c|c|c|}
\hline Feature & $\begin{array}{c}\text { Functionality } \\
\text { Social Care } \\
\text { Application }\end{array}$ & $\begin{array}{c}\text { App } \\
\boldsymbol{A}\end{array}$ & $\begin{array}{c}\boldsymbol{A p p} \\
\boldsymbol{B}\end{array}$ & $\begin{array}{c}\text { App } \\
\boldsymbol{C}\end{array}$ & $\begin{array}{c}\text { App } \\
\boldsymbol{D}\end{array}$ \\
\hline $\begin{array}{c}\text { Search } \\
\text { Search })\end{array}$ & Service & $\checkmark$ & $\checkmark$ & - & - \\
\hline $\begin{array}{c}\text { Worker } \\
\text { Performance } \\
\text { Rating }\end{array}$ & Reputation & $\checkmark$ & - & - & - \\
\hline $\begin{array}{c}\text { Application } \\
\text { Rating }\end{array}$ & Reputation & - & $\checkmark$ & $\checkmark$ & $\checkmark$ \\
\hline Best Dealer & Reputation & $\checkmark$ & & & \\
\hline $\begin{array}{c}\text { Tips \& } \\
\text { Articles }\end{array}$ & Sharing & $\checkmark$ & $\checkmark$ & $\checkmark$ & $\checkmark$ \\
\hline $\begin{array}{c}\text { Social } \\
\text { Media }\end{array}$ & Sharing & - & - & - & - \\
\hline
\end{tabular}

\subsection{Mapping of Features to E-marketplace Social Care}

\section{Functionality}

The following table is features mapping based on category or functionality attributes in e-marketplace social care.

Table 2: Mapping of Features to E-marketplace Functionality

\begin{tabular}{|l|l|c|c|c|}
\hline Identity & Conversation & $\begin{array}{c}\text { Information } \\
\text { \& Service }\end{array}$ & Reputation & Sharing \\
\hline Photo & $\begin{array}{l}\text { Chat } \\
\text { Assistance } \\
\text { Services }\end{array}$ & $\begin{array}{c}\text { Special } \\
\text { Request }\end{array}$ & $\begin{array}{c}\text { Worker } \\
\text { Performance } \\
\text { Rating }\end{array}$ & $\begin{array}{c}\text { Tips \& } \\
\text { Articles }\end{array}$ \\
\hline Sign Up & Testimonial & $\begin{array}{c}\text { List of } \\
\text { Services \& } \\
\text { Detail of } \\
\text { Services }\end{array}$ & $\begin{array}{c}\text { Application } \\
\text { Rating }\end{array}$ & $\begin{array}{c}\text { Social } \\
\text { Media }\end{array}$ \\
\hline $\begin{array}{l}\text { Account } \\
\text { Profile }\end{array}$ & Like & $\begin{array}{c}\text { Order and } \\
\text { Order } \\
\text { History }\end{array}$ & Best Dealer & \\
\hline $\begin{array}{l}\text { Forgot } \\
\text { Password }\end{array}$ & & Search & & \\
\hline \multicolumn{1}{|c|}{4} & 3 & 4 & 3 & 2 \\
\hline
\end{tabular}

\subsection{Orthoplan}

In obtaining the minimum number of combinations stimuli that orthogonal respondent must evaluate, the orthoplan process or orthogonal array is used. Orthogonal is the absence of correlation between levels on an attribute [11]. Based on the orthoplan formula, the result of the calculation is:

N_Identity * N_Conversation * N_Information\&Service * N_Reputation * N_Sharing

Orthoplan $=4 * 3 * 4 * 3 * 2=288$ combination. The result of each component multiplication is 288 combination. Therefore, a randomized orthoplan was conduct in this study because it was not possible to test 288 combination.

The following is a table of random orthoplan results generated by the system using SPSS version 23 used in the questionnaire.

Table 3: Result of Random Feature Based on Orthoplan

\begin{tabular}{|l|l|l|l|l|}
\hline Identity & Conversation & $\begin{array}{l}\text { Information } \\
\text { \& Service }\end{array}$ & Reputation & Sharing \\
\hline Photo & $\begin{array}{l}\text { Chat } \\
\text { Assistance } \\
\text { Services }\end{array}$ & $\begin{array}{l}\text { Special } \\
\text { Request }\end{array}$ & $\begin{array}{l}\text { Application } \\
\text { Rating }\end{array}$ & $\begin{array}{l}\text { Social } \\
\text { Media }\end{array}$ \\
\hline
\end{tabular}

\begin{tabular}{|c|c|c|c|c|}
\hline Identity & Conversation & $\begin{array}{l}\text { Information } \\
\text { \& Service }\end{array}$ & Reputation & Sharing \\
\hline $\begin{array}{l}\text { Forgot } \\
\text { Password }\end{array}$ & $\begin{array}{l}\text { Chat } \\
\text { Assistance } \\
\text { Services }\end{array}$ & $\begin{array}{l}\text { Order and } \\
\text { Order } \\
\text { History }\end{array}$ & $\begin{array}{l}\text { Application } \\
\text { Rating }\end{array}$ & $\begin{array}{l}\text { Tips \& } \\
\text { Articles }\end{array}$ \\
\hline Photo & $\begin{array}{l}\text { Chat } \\
\text { Assistance } \\
\text { Services }\end{array}$ & $\begin{array}{l}\text { Special } \\
\text { Request }\end{array}$ & $\begin{array}{l}\text { Worker } \\
\text { Performance } \\
\text { Rating }\end{array}$ & $\begin{array}{l}\text { Social } \\
\text { Media }\end{array}$ \\
\hline Photo & Like & $\begin{array}{l}\text { Special } \\
\text { Request }\end{array}$ & $\begin{array}{l}\text { Application } \\
\text { Rating }\end{array}$ & $\begin{array}{l}\text { Social } \\
\text { Media } \\
\end{array}$ \\
\hline $\begin{array}{l}\text { Account } \\
\text { Profile }\end{array}$ & $\begin{array}{l}\text { Chat } \\
\text { Assistance } \\
\text { Services }\end{array}$ & $\begin{array}{l}\text { List of } \\
\text { Services \& } \\
\text { Detail of } \\
\text { Services } \\
\end{array}$ & $\begin{array}{l}\text { Worker } \\
\text { Performance } \\
\text { Rating }\end{array}$ & $\begin{array}{l}\text { Tips \& } \\
\text { Articles }\end{array}$ \\
\hline Photo & Testimonial & $\begin{array}{l}\text { Order and } \\
\text { Order } \\
\text { History } \\
\end{array}$ & $\begin{array}{l}\text { Worker } \\
\text { Performance } \\
\text { Rating }\end{array}$ & $\begin{array}{l}\text { Tips \& } \\
\text { Articles }\end{array}$ \\
\hline Sign Up & $\begin{array}{l}\text { Chat } \\
\text { Assistance } \\
\text { Services }\end{array}$ & $\begin{array}{l}\text { List of } \\
\text { Services \& } \\
\text { Detail of } \\
\text { Services }\end{array}$ & $\begin{array}{l}\text { Application } \\
\text { Rating }\end{array}$ & $\begin{array}{l}\text { Tips \& } \\
\text { Articles }\end{array}$ \\
\hline $\begin{array}{l}\text { Account } \\
\text { Profile }\end{array}$ & Testimonial & $\begin{array}{l}\text { Special } \\
\text { Request }\end{array}$ & $\begin{array}{l}\text { Worker } \\
\text { Performance } \\
\text { Rating }\end{array}$ & $\begin{array}{l}\text { Tips \& } \\
\text { Articles }\end{array}$ \\
\hline Photo & Like & $\begin{array}{l}\text { List of } \\
\text { Services \& } \\
\text { Detail of } \\
\text { Services } \\
\end{array}$ & Best Dealer & $\begin{array}{l}\text { Tips \& } \\
\text { Articles }\end{array}$ \\
\hline $\begin{array}{l}\text { Account } \\
\text { Profile }\end{array}$ & $\begin{array}{l}\text { Chat } \\
\text { Assistance } \\
\text { Services }\end{array}$ & $\begin{array}{l}\text { Order and } \\
\text { Order } \\
\text { History }\end{array}$ & Best Dealer & $\begin{array}{l}\text { Social } \\
\text { Media }\end{array}$ \\
\hline $\begin{array}{l}\text { Forgot } \\
\text { Password }\end{array}$ & $\begin{array}{l}\text { Chat } \\
\text { Assistance } \\
\text { Services }\end{array}$ & Search & $\begin{array}{l}\text { Worker } \\
\text { Performance } \\
\text { Rating }\end{array}$ & $\begin{array}{l}\text { Tips \& } \\
\text { Articles }\end{array}$ \\
\hline Photo & $\begin{array}{l}\text { Chat } \\
\text { Assistance } \\
\text { Services }\end{array}$ & Search & Best Dealer & $\begin{array}{l}\text { Tips \& } \\
\text { Articles }\end{array}$ \\
\hline Sign Up & Like & $\begin{array}{l}\text { Order and } \\
\text { Order } \\
\text { History }\end{array}$ & $\begin{array}{l}\text { Worker } \\
\text { Performance } \\
\text { Rating }\end{array}$ & $\begin{array}{l}\text { Social } \\
\text { Media }\end{array}$ \\
\hline $\begin{array}{l}\text { Account } \\
\text { Profile }\end{array}$ & Like & Search & $\begin{array}{l}\text { Application } \\
\text { Rating }\end{array}$ & $\begin{array}{l}\text { Tips \& } \\
\text { Articles } \\
\end{array}$ \\
\hline $\begin{array}{l}\text { Forgot } \\
\text { Password }\end{array}$ & Testimonial & $\begin{array}{l}\text { List of } \\
\text { Services \& } \\
\text { Detail of } \\
\text { Services } \\
\end{array}$ & $\begin{array}{l}\text { Application } \\
\text { Rating }\end{array}$ & $\begin{array}{l}\text { Social } \\
\text { Media }\end{array}$ \\
\hline Sign Up & Testimonial & Search & $\begin{array}{l}\text { Worker } \\
\text { Performance } \\
\text { Rating }\end{array}$ & $\begin{array}{l}\text { Social } \\
\text { Media }\end{array}$ \\
\hline Sign Up & Testimonial & $\begin{array}{l}\text { Special } \\
\text { Request }\end{array}$ & Best Dealer & $\begin{array}{l}\text { Tips \& } \\
\text { Articles } \\
\end{array}$ \\
\hline Photo & Testimonial & $\begin{array}{l}\text { List of } \\
\text { Services \& } \\
\text { Detail of } \\
\text { Services } \\
\end{array}$ & $\begin{array}{l}\text { Worker } \\
\text { Performance } \\
\text { Rating }\end{array}$ & $\begin{array}{l}\text { Tips \& } \\
\text { Articles }\end{array}$ \\
\hline Photo & $\begin{array}{l}\text { Chat } \\
\text { Assistance } \\
\text { Services }\end{array}$ & $\begin{array}{l}\text { Special } \\
\text { Request }\end{array}$ & $\begin{array}{l}\text { Worker } \\
\text { Performance } \\
\text { Rating }\end{array}$ & $\begin{array}{l}\text { Tips \& } \\
\text { Articles }\end{array}$ \\
\hline $\begin{array}{l}\text { Forgot } \\
\text { Password }\end{array}$ & Like & $\begin{array}{l}\text { Special } \\
\text { Request }\end{array}$ & $\begin{array}{l}\text { Worker } \\
\text { Performance } \\
\text { Rating }\end{array}$ & $\begin{array}{l}\text { Tips \& } \\
\text { Articles }\end{array}$ \\
\hline Sign Up & $\begin{array}{l}\text { Services } \\
\text { Assistance } \\
\text { Chat }\end{array}$ & $\begin{array}{l}\text { Special } \\
\text { Request }\end{array}$ & $\begin{array}{l}\text { Application } \\
\text { Rating }\end{array}$ & $\begin{array}{l}\text { Tips \& } \\
\text { Articles }\end{array}$ \\
\hline
\end{tabular}


E. Angelina et al. / Advances in Science, Technology and Engineering Systems Journal Vol. 5, No. 6, 1593-1597 (2020)

\begin{tabular}{|l|l|l|l|l|}
\hline Identity & Conversation & $\begin{array}{l}\text { Information } \\
\text { \& Service }\end{array}$ & Reputation & Sharing \\
\hline $\begin{array}{l}\text { Account } \\
\text { Profile }\end{array}$ & Testimonial & $\begin{array}{l}\text { Special } \\
\text { Request }\end{array}$ & $\begin{array}{l}\text { Application } \\
\text { Rating }\end{array}$ & $\begin{array}{l}\text { Tips \& } \\
\text { Articles }\end{array}$ \\
\hline Photo & Testimonial & $\begin{array}{l}\text { Order and } \\
\text { Order } \\
\text { History }\end{array}$ & $\begin{array}{l}\text { Application } \\
\text { Rating }\end{array}$ & $\begin{array}{l}\text { Tips \& } \\
\text { Articles }\end{array}$ \\
\hline $\begin{array}{l}\text { Forgot } \\
\text { Password }\end{array}$ & Testimonial & $\begin{array}{l}\text { Special } \\
\text { Request }\end{array}$ & Best Dealer & $\begin{array}{l}\text { Social } \\
\text { Media }\end{array}$ \\
\hline Photo & Testimonial & Search & $\begin{array}{l}\text { Application } \\
\text { Rating }\end{array}$ & $\begin{array}{l}\text { Social } \\
\text { Media }\end{array}$ \\
\hline
\end{tabular}

Based on the random orthoplan result table above, the user must rate 1-25 of each row in the table, where the user can only fill in 1 rating number on each row and this number appear once on each line.

\subsection{E-marketplace Social Care Feature Preferences}

The following is the result of e-marketplace social care feature preferences based on conjoint analysis using SPSS version 23:

Table 4: The Result of E-marketplace Social Care Feature Preferences

\begin{tabular}{|c|c|c|c|}
\hline Functionality & Features & $\begin{array}{c}\text { Importance } \\
\text { Value }\end{array}$ & $\begin{array}{c}\text { Utility } \\
\text { Estimate }\end{array}$ \\
\hline \multirow[t]{4}{*}{ Identity } & Photo & \multirow{4}{*}{21.996} & 0.355 \\
\hline & Sign Up & & -0.013 \\
\hline & Account Profile & & -0.080 \\
\hline & $\begin{array}{l}\text { Forgot } \\
\text { Password }\end{array}$ & & -0.262 \\
\hline \multirow[t]{3}{*}{ Conversation } & $\begin{array}{l}\text { Service } \\
\text { Assistance Chat }\end{array}$ & \multirow{3}{*}{25.026} & 0.146 \\
\hline & Testimonial & & -0.058 \\
\hline & Like & & -0.089 \\
\hline \multirow{4}{*}{$\begin{array}{l}\text { Information } \\
\text { \& Service }\end{array}$} & Special Request & \multirow{4}{*}{24.402} & 0.302 \\
\hline & $\begin{array}{l}\text { List of Services } \\
\& \text { Detail of } \\
\text { Services }\end{array}$ & & -0.068 \\
\hline & $\begin{array}{l}\text { Order \& Order } \\
\text { History }\end{array}$ & & -0.028 \\
\hline & Search & & -0.205 \\
\hline \multirow[t]{3}{*}{ Reputation } & $\begin{array}{l}\text { Worker } \\
\text { Performance } \\
\text { Rating }\end{array}$ & \multirow{3}{*}{16.351} & 0.027 \\
\hline & $\begin{array}{l}\text { Application } \\
\text { Rating }\end{array}$ & & 0.303 \\
\hline & Best Dealer & & -0.330 \\
\hline \multirow[t]{2}{*}{ Sharing } & Tips \& Articles & \multirow{2}{*}{12.226} & 0.011 \\
\hline & Social Media & & -0.011 \\
\hline \multicolumn{3}{|l|}{ (Constant) } & 12.906 \\
\hline \multirow[t]{3}{*}{ Correlations $^{\mathrm{a}}$} & & Value & Sig. \\
\hline & Pearson's R & .558 & .002 \\
\hline & Kendall's tau & .320 & .012 \\
\hline
\end{tabular}

Based on the result of conjoint analysis of 521 respondents on the attributes of social care functionality in the table above, the conclusions are as follows:

1. In the Identity section, based on the result of statistical test can be summed that the respondent prefers the form of feature photo. It can be seen from the utility value which shows a positive result with 0.355 for the photo feature. Meanwhile the Sign Up, Account Profile and Forgot

Password have a negative utility value. It can be seen from the utility value which shows a negative result with -0.013 for Sign Up, -0.089 for Account Profile feature, -0.262 for Forgot Password feature.

2. In the Conversation section, based on the result of statistical test can be summed that the respondent prefers the form of Services Assistance Chat feature. It can be seen from the utility value which shows a positive result with 0.146 for the Services Assistance Chat feature. Meanwhile the Testimonial and Like features have a negative utility value. It can be seen from the utility value which shows a negative result with -0.058 for testimonial feature and -0.089 for Like feature.

3. In the Information and Service section, based on the result of statistical test can be summed that the respondent prefers the form of Special Request feature. It can be seen from the utility value which shows a positive result with 0.302 for the Special Request feature. Meanwhile the List of Service \& Service Detail, Order \& Order History and Search feature have a negative utility value. It can be seen from the utility value which shows a negative result with -0.068 for List of Service \& Service Detail feature, -0.028 Order \& Order History feature and -0.205 for Search feature.

4. In the Reputation section, based on the result of statistical test can be summed that the respondent prefers the form of Worker Performance Rating and Application Rating feature. It can be seen from the utility value which shows a positive result with 0.027 for the Worker Performance Rating feature and 0.303 for Application Rating feature. Meanwhile the Best Dealer feature has a negative utility value -0.330 .

5. In the Sharing section, based on the result of statistical test can be summed that the respondent prefers the form of List of Tips \& Articles feature. It can be seen from the utility value which shows a positive result with 0.011 for the List of Tips $\&$ Articles feature. Meanwhile the social media feature has a negative utility value -0.011 .

\subsection{Conjoint Analysis Hypothesis Test on Media Social Functionality}

By using the predictive accuracy conjoint test can be measured using Pearson's R Correlation. In this study, the Pearson's R value was 0.558 with a significance level 0.002 and Kendall's Tau correlation showed a value 0.320 with a significance level 0.012 . This indicates a correlation between the respondent opinion patterns (estimates part-worth) and actual preferences. For each respondent preference towards factors on social care functionality which is divided into five parts, namely Identity, Conversation, Information and Services, Reputation, Sharing.

$\mathrm{H}_{0}=$ Estimated opinion patterns of respondents differ greatly $\mathrm{H}_{1}=$ Estimated opinion patterns of respondents do not differ that much

Based on the result, it can be concluded that $\mathrm{H}_{0}$ is rejected and $\mathrm{H}_{1}$ is accepted, because:

- The significance level of Pearson's $\mathrm{R}=0.002<0.05$

- The significance level of Kendall's Tau $=0.012<0.05$ 
The result of conjoint analysis shows that the estimates of respondent opinion pattern (estimates part-worth) do not much differ from the respondent actual opinion (actual preferences) on the stimuli process.

\section{Conclusion}

The 6 features that have a positive value from the results of conjoint analysis, namely Photo, Chat Assistance Services, Special Requests, Worker Performance Ratings, Application Ratings, and Tips \& Articles, are 6 features that are the main feature preferences in the social e-marketplace system. care and at the same time these 6 features answer the first research question.

As well as to answer the second research question, based on the results of the conjoint analysis can prove that the approximate opinion patterns of respondents are not much different from the actual opinion of the respondent which can be seen from the feature rating given by users as a result of user satisfaction in using the features, and it can answer the problem with determining feature preferences can help increase the intensity of the use of the e-marketplace social care system.

\section{Limitation and Future Research}

This research is limited to the feature preferences of the emarketplace social care application system which users assess as a feature rating on the random orthoplan results which later on these features will be maintained and used in the other emarketplace social care system development.

Further research activity needs to be reassessed in terms of the service capability in meeting user needs, from user satisfaction in using this type of service on the e-marketplace social care so that companies can improve the quality of services offered.

\section{References}

[1] L. Åberg, T. Pham, J. Tomsfelt, " A Study about E-loyalty using the Bagozzi framework," 2013.

[2] G.L. Polites, C.K. Williams, E. Karahanna, L. Seligman, "A Theoretical Framework for Consumer E-Satisfaction and Site Stickiness: An Evaluation in the Context of Online Hotel Reservations," Journal of Organizational Computing and Electronic Commerce, 2012, doi:10.1080/10919392.2012.642242.

[3] S.H. Hsu, W.H. Chen, J.T. Hsueh, "Application of customer satisfaction study to derive customer knowledge," Total Quality Management and Business Excellence, 17(4), 439-454, 2006, doi: $10.1080 / 14783360500528197$.

[4] E. Christiaanse, M.L. Markus, "Participation in collaboration electronic marketplaces," in Proceedings of the 36th Annual Hawaii International Conference on System Sciences, HICSS-2003, 2003, doi:10.1109/HICSS.2003.1174383.

[5] S. Wang, N.P. Archer, "Electronic marketplace definition and classification: Literature review and clarifications," Enterprise Information Systems, 2007, doi:10.1080/17517570601088380.

[6] M.M. Cruz-Cunha, I. Miranda, N. Lopes, R. Simoes, "An e-marketplace of healthcare and social care services: The perceived interest," Learning Organization, 2013, doi:10.1108/TLO-10-2013-0055.

[7] G. Armstrong, P. Kotler, M. Harker, R. Brennan, "Marketing: An introduction," 2010.

[8] P. Silayoi, M. Speece, "The importance of packaging attributes: A conjoint analysis approach," European Journal of Marketing, 2007, doi:10.1108/03090560710821279.

[9] J.F. Hair, R.E. Anderson, R.L. Tatham, W.C. Black, Multivariate Data Analysis, Multivariate Data Analysis, 2018.

[10] H.I. Ghozali, Aplikasi Analisis Multivariate dengan Program IBM SPSS 19, 5th ed., Undip, Semarang, 2005.
[11] R.L. Hair, J. F., Black, W. C., Babin, B. J., Anderson, R. E., \& Tatham, Multivariate Data Analysis, 2009. 